\title{
Aggressive behaviors in the psychiatric emergency service
}

\author{
This article was published in the following Dove Press journal: \\ Open Access Emergency Medicine \\ 8 March 201I \\ Number of times this article has been viewed
}

\section{Yves Chaput' \\ Lucie Beaulieu ${ }^{2}$ \\ Michel Paradis ${ }^{3}$ \\ Edith Labonté}

'Department of Psychiatry, McGill University, Montreal (presently in private practice); ${ }^{2}$ Department of Psychiatry, Haut Richelieu Hospital, Saint-Jean-sur-Richelieu, Quebec; ${ }^{3}$ Department of Psychiatry, University of Montreal, Montreal; ${ }^{4}$ Department of Psychiatry, Laval University, Quebec, Canada
Correspondence: Yves Chaput 365 rue Normand, Suite 230, Saint-Jean-sur-Richelieu, Quebec J3A IT6, Canada Tel +I 4503599535

Fax + I 4503590712

Email yveschaput@bellnet.ca
Introduction: Studies of aggressive behaviors in a nonforensic mental health setting have focused primarily on the inpatient ward and, on event prediction, using behavior-based clinical rating scales. Few studies have specifically targeted aggressive behaviors in the psychiatric emergency service or determined whether assessing the demographic and clinical characteristics of such patients might prove useful for their more rapid identification.

Methods: We used a prospectively acquired database of over 20,900 visits to four services in the province of Quebec, Canada, over a two-year period from September 2002 onwards. A maximum of 72 variables could be acquired per visit. Visits with aggression (any verbally or physically intimidating behavior), both present and past, were tagged. Binary logistic regressions and cross-tabulations were used to determine whether the profile of a variable differed in visits with aggression from those without aggression.

Results: About $7 \%$ of visits were marked by current aggression (verbal $49 \%$, physical $12 \%$, verbal and physical 39\%). Including visits with a "past only" history of aggression increased this number to $20 \%$. Variables associated with aggression were gender (male), marital status (single/separated), education (high school or less), employment (none), judicial history (any type), substance abuse (prior or active), medication compliance (poor), type of arrival to psychiatric emergency services (involuntary, police, judiciary, landlord), reason for referral (behavioral dyscontrol), diagnosis (less frequent in anxiety disorders), and outcome (more frequently placed under observation or admitted).

Conclusion: Our results suggest that many state-independent variables are associated with aggressive behaviors in the psychiatric emergency service. Although their sum may not add up to a specific patient profile, they can nevertheless be useful in service planning, being easily integrated alongside state-dependent rating scales in a triage and/or observation instrument for daily use in the psychiatric emergency service.

Keywords: psychiatric emergency service, aggressive behaviors, epidemiology

\section{Introduction}

Disruptive acts of violence in a nonforensic mental health setting can take an emotional toll on both staff and patients. Although widespread and observable in almost all clinical settings, ${ }^{1-5}$ aggressive behaviors have been most frequently studied in inpatients. ${ }^{1,6-9}$ Generally, assaultive patients have been described as being either older schizophrenic males or younger, gender nonspecific patients with personality disorders. ${ }^{1,10}$ Both types of profiles are often associated with a history of comorbid substance abuse, as well as a history of violence towards others. . $^{1,4,7,8}$

The psychiatric emergency service (PES) is a major hub in the mental health care delivery system. In its most elementary form, it consists of the interaction between a 
psychiatrist (or health care professional) and a patient within the framework of a crisis situation. Studies specifically targeting aggressive behaviors in the PES have been fewer in number, as well as less consistent in providing definitive clinical profiles. Estimates of their frequency in the PES have ranged from $6 \%$ to $17 \%$ of all visits. ${ }^{10-12}$ Although an acute psychotic state (regardless of its origin) and recent aggressive behaviors prior to a PES visit appear to be associated, ${ }^{10,11,13-15}$ variables such as age, gender, or substance abuse have shown less consistency. ${ }^{10,13,14}$ This inconsistency may not only be due to the relatively small number of available PES studies on this topic, but also to the generally broader PES diagnostic profile (compared with the inpatient setting) and to a host of variables (some sociodemographic, some even environmental) that do not have inpatient counterparts. ${ }^{4,16-18}$ Matching aggressive behaviors in the PES to a consistent patient profile was the primary objective of our study. Such a finding may prove useful at the triage level, especially if combined with more "state or emotional-based" rating instruments typically used to assess such high-risk behaviors. ${ }^{6,19}$ We pursued this objective by using a prospectively acquired database of over 20,900 PES visits made to four PES in the province of Quebec, Canada, over a two-year period.

\section{Methods}

Data collection was as previously described. ${ }^{16,17,20}$ Clinical and demographic data were obtained from patients 18 years of age and older visiting four major PES between September 1, 2002 and August 31, 2004. Two of these services were in the city of Montreal in Quebec, Canada. The first was in a downtown university teaching hospital and the second was within a university affiliated psychiatric institute near the downtown core. This latter PES did not have a medical emergency department or prior medical triage, and largely functioned as a "walk-in clinic". Each PES in metropolitan Montreal is assigned a geographic catchment area, and citizens within it are obliged to seek acute psychiatric care at that service only. Approximately $4.8 \%$ of all patients who underwent triage in the emergency department of the general hospital PES were referred for a psychiatric assessment. Two sites were in cities other than Montreal. One was in Quebec City (300 km east of Montreal, with approximately 500,000 citizens) and the other in Saint-Jean-sur-Richelieu (40 km south of Montreal, with approximately 90,000 citizens). This latter site differed from the others by not having an observation area with short-term beds. ${ }^{20}$
A maximum of 72 variables could be acquired per visit. The main table contained administrative variables (eg, chart number, name, and gender). Linked tables contained variables pertinent to the consultation process, eg, date and time of arrival, reasons for the referral, psychosocial stressors prior to the visit, referral source, ethnicity, employment, residential status, disposition, and DSM-IV (Diagnostic and Statistical Manual of Mental Disorders, 4th Edition) diagnoses (three per visit). Qualitative data were also acquired, including a subjective rating by the nursing staff of the level of aggression upon arrival to the PES (verbal aggression, physical aggression, both, none at all). Staff responses, eg, verbal pacification, isolation without restraints, or isolation plus restraints, were also categorized. All variables in the database were listed in a paper format, which was used as the primary triage instrument for patients visiting the four services during the two-year period. The completed forms were forwarded to the principal investigator on a weekly basis for data entry.

Many strategies were used in order to minimize diagnostic uncertainty. First, because over $60 \%$ of PES visits have been shown to occur within the daytime hours, ${ }^{16}$ only services that were covered on weekdays by experienced, regular daytime psychiatric staff with over five years' experience in the PES setting were included. None of the four sites provided midnight to 7 am assessments. Patients referred from the emergency department during this time period were kept in the psychiatric observation area for assessment in the morning. As such, during weekdays, well over $70 \%-80 \%$ of patients were assessed by the regular PES staff. Most staff obtained their medical and specialty training at one the four medical faculties in the province, and thus shared a common set of methodological, ethical, and cultural standards. Second, diagnoses made using DSM-IV guidelines during nonstructured clinical interviews were obtained either directly from staff after patient assessment or from the patient's chart. Third, diagnoses were grouped into broad categories, which included "none", "adjustment", "anxiety", "personality", "affective", "schizophrenia", "psychosis not otherwise specified", "substance abuse", and "organic mental disorders". Fourth, for patients with two or more visits, a "most probable" primary diagnosis was attributed, which was the diagnosis most frequently given. The second most frequently attributed diagnosis was retained as comorbidity. Fifth, as previously reported, ${ }^{16}$ from $65 \%-80 \%$ of frequent users at all sites were at one point in time under multidisciplinary outpatient care and, as such, any diagnostic uncertainty could be clarified with the treating team. 


\section{Statistical analysis}

Data was analyzed using Systat Version 13. The dataset for this study comprised 13,592 patients making 20,963 visits. Most variables were of the nominal type (binary or with multiple categories). Preliminary analyses consisted of constructing contingency tables where the binary dependent variable (presence or absence of violence) was tabulated with an independent predictor variable (eg, gender). If the $P$ values for the Pearson Chi-square and the likelihood ratio Chi-square were $<0.05$, the data were retabulated using a goodness of fit model to determine if the profile of the dependent variable differed significantly from the independent predictor variable using the distribution ratios of visits marked by aggression as the expected frequencies. Only data for which the two procedures were significant are presented in the Results section. In addition, binary logistic regressions and their resulting odds ratios (OR, with 95\% confidence intervals [CI]) were used to determine the strength and direction of an association between the dependent and independent predictor variable.

This study was approved by the scientific subcommittees of the institutional review boards at all sites other than at the psychiatric institute, and was exempted from full review. Full institutional review board approval was required and obtained at the psychiatric institute site.

\section{Results}

Overall, $19.7 \%$ of all visits (4134 of 20,963 in total made during the observation period) were tagged as having either a present or a past history of aggressive behaviors in the PES. In $4 \%$ of visits (167 of 4134) marked by aggressive behaviors, a "current or past" specification was not tagged, and these were dropped from any further statistical analyses. Of the remaining 3967 visits, $22.4 \%(\mathrm{n}=888)$ were tagged as de novo "current" cases, $16.5 \%(\mathrm{n}=654)$ as "current" but also having a "past" history of aggressive behaviors in the PES, and in $61 \%(\mathrm{n}=2425)$ of cases there was a "past" history of aggressive behaviors in the PES only. Unless stated otherwise, most statistical procedures, with the exception of those pertaining to gender and age, were performed on patients presenting with current aggressive behaviors ( $n=1542$ ) who constituted $7.4 \%$ of all PES visits.

In visits from patients with a current history of aggressive behaviors, the nature of the aggression was documented in 1129 cases, and was verbal in $49 \%$, physical in $12 \%$, and both verbal and physical in 39\%. The staff response to aggressive behaviors was documented in 1062 incidents, and comprised isolation/seclusion (23\%), physical restraints (7\%), both $(21 \%)$, and in $50 \%$ of cases staff responded by using a verbal pacification approach.

\section{Sociodemographic variables}

Gender differences were clearly in evidence. The male/ female percentage distribution of patients without aggressive behaviors was 49/51 $(n=16,829)$, whereas that of visits from patients with a current and/or past history of aggressive behaviors was $60 / 40(n=3967)$. A male/female percentage difference was evident in all groups of visits from patients with aggressive behaviors (current 56/44, current and past $62 / 38$, past only 61/39). Overall, males were 1.57 times more likely to have a current and/or past history of aggressive behaviors than women $(P<0.001$, CI 1.46-1.68)

Age as a predictor variable was then examined (Figure 1). There was a significant, albeit very slight, decrease in the probability $(P<0.001$, OR 0.938 , CI $0.927-0.950)$ of aggressive behaviors (combined group) with increasing age. This was so whether age was in 5-year or 10-year intervals (from age 20 years onwards) or by using a binary variable, ie, dividing patients into two age groups, one 20-40 years and the other $\geq 41$ years. The OR was still significant with gender inserted as a binary interactive variable, although it was slightly less robust $(P<0.001$, OR 0.941, CI 0.929-0.953).

Marital, educational, employment, and residential status were examined next. A current history of aggressive behaviors $(\mathrm{n}=1451)$ was more likely to be seen in visits from patients who were single $(P<0.001$, OR 1.43, CI 1.29-1.60) or divorced/separated $(P<0.001$, OR 1.39, CI 1.20-1.61). Patients with a high school (or lower) educational status were 1.7 times more likely to present to the PES with aggressive

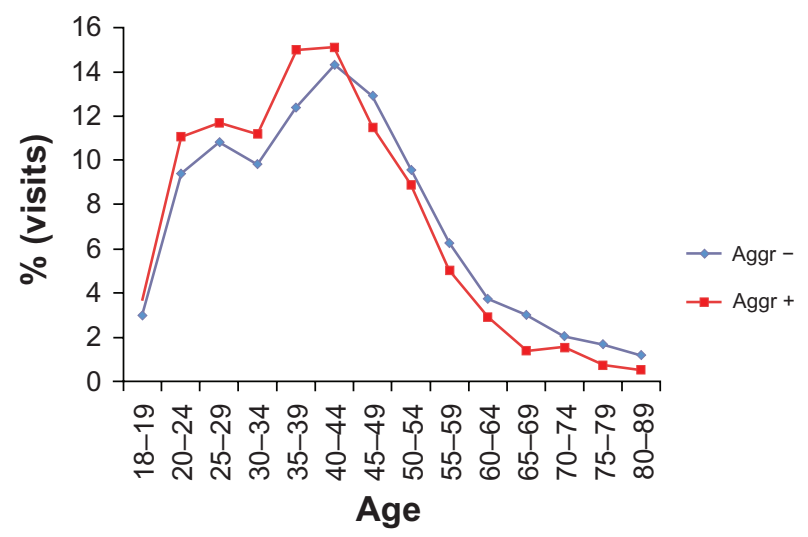

Figure I Age profile of visits with and without aggression. Summing up all data points of either curve totals $100 \%$. Visits with aggression $(n=4050$, combined group, including those with a current history of aggression and those with a past history) and visits without aggression $(n=16,693)$.

Abbreviations: Aggr+, with aggression; Aggr-, without aggression. 
behaviors than those with a college or university background $(P<0.001$, CI 1.48-2.00). Those who were unemployed were 1.74 times more likely to present with aggressive behaviors compared with those in either full-time or part-time employment ( $P<0.001$, CI 1.55-1.95). Residential status was divided into those who rented, were homeowners, were in some kind of residence (mental health or not), and the homeless (including those in temporary shelters). There was a tendency for those in residential living conditions to have more visits with aggression (Pearson Chi-square $P<0.001$, two-way tables with either of the two aggression groups as the dependent variable). However, overall, the logistic regression for this model was not significant. A history of any type of prior illegal activity leading to an arrest was assessed in 10,420 visits (928 with aggression and 9492 without aggression) and, as expected, was much more likely in patients with than without aggression $(P<0.001$, OR 3.15, CI 2.74-3.62).

\section{Arrival to PES}

Time of arrival to the PES, as assessed by the number of visits/four-hour periods beginning at midnight, did not differentiate visits marked by aggression from those without aggression, although there was a tendency for those with aggression to congregate between noon to $8 \mathrm{pm}$. Frequency of use was examined by dividing patients into groups making one $(n=9826), 2-3(n=2540), 4-10(n=646)$, or $\geq 11$ visits $(n=58)$. These anchor points have been shown to result in distinct diagnostic subgroups of PES users. ${ }^{16,17,21}$ No significant relationship could be found between frequency of PES use and visits from patients with a current history of aggression. However, using the combined group $(n=3967)$ as the dependent variable, there was a slightly increased likelihood ( $P<0.001$, OR 1.18, CI 1.095-1.269) of being in a frequent PES user group.

Types of arrival to the PES were then examined. Overall, involuntary referrals (regardless of the source) were significantly more likely ( $P<0.001$, OR 4.14 , CI 3.69-4.64) to have a current history of aggressive behaviors upon arrival to the PES ( $n=1439$ for visits from patients with a current history of aggression). The OR for the 10 primary sources of referral are shown in Figure 2.

\section{Substance abuse}

A history (past or current) of substance abuse was more frequently found in visits from patients with a current history of aggression $(P<0.001$, OR 1.67, CI 1.48-1.87,

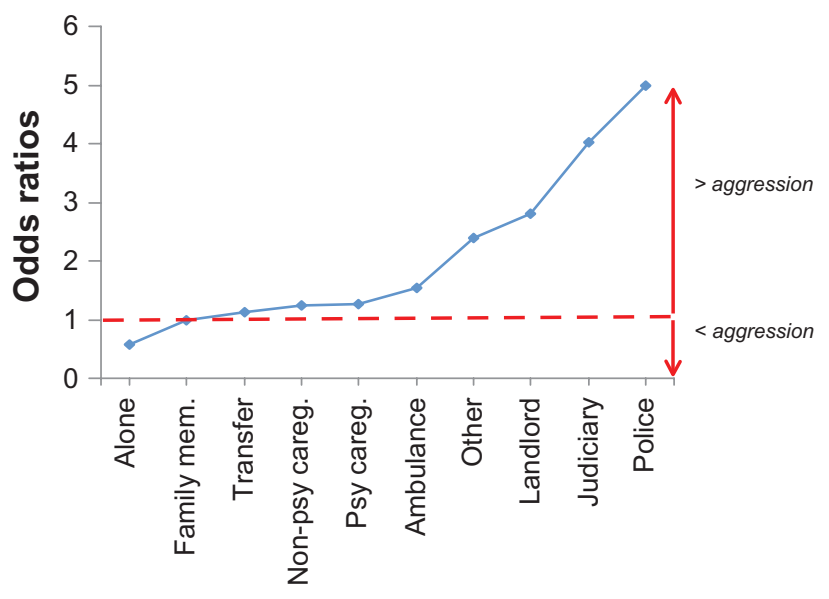

Figure 2 Odds ratios (OR) for the various types of arrivals to the psychiatric emergency services for visits with a current history of aggression $(n=1476)$ versus those without $(n=16,003)$. Alone (OR 0.58, $P<0.001$, confidence interval $[\mathrm{Cl}]$ 0.44-0.78, $\mathrm{n}=193$ ), with a family member (OR $0.98, P=0.87$, $\mathrm{Cl} 0.74-1.29, \mathrm{n}=276$ ), transfer (from another psychiatric emergency service, OR I.I6, $P=0.43, \mathrm{Cl} 0.8 \mathrm{I}-\mathrm{I} .27, \mathrm{n}=59$ ), with a nonpsychiatric caregiver (OR I.36, $P=0.2 \mathrm{I}, \mathrm{Cl} 0.84-2.20, \mathrm{n}=24)$, with a psychiatric caregiver $(\mathrm{OR} \mathrm{I} .32, P=0.18$, $\mathrm{Cl} 0.89-2.00, \mathrm{n}=40$ ), ambulance (OR I.6, $P<0.01, \mathrm{Cl} 1.22-2.10, \mathrm{n}=37 \mathrm{I}$ ), landlord (OR 2.8, $P<0.00 \mathrm{I}, \mathrm{Cl} 1.78-4.40, \mathrm{n}=32$ ), judiciary (court referrals, OR 4.0, $P<0.00$ I, Cl 2.2-7.5, $\mathrm{n}=\mathrm{I}$ ), police (OR 5.0, $P<0.00 \mathrm{I}, \mathrm{Cl} 3.8-6.6, \mathrm{n}=406$ ), other (all sources other than those listed, OR 2.6, $P<0.00$ I, Cl I.8-3.7, $\mathrm{n}=59$ ).

Abbreviations: Nonpsy careg, nonpsychiatric caregiver; Psy careg, psychiatric caregiver; Family mem, family member.

$\mathrm{n}=1390$ visits) as well as recent (within 24 hours) alcohol or drug consumption $(P<0.001$, OR 1.75, CI 1.55-1.99, $\mathrm{n}=1542$ visits). However, the substance of choice (alcohol, benzodiazepines, cannabis, hallucinogens, amphetamines, cocaine, opiates) was similar for visits with and without aggressive behaviors. In 4895 visits ( 505 where aggression was current, 4390 without) nursing staff members were asked to provide a subjective opinion as to whether substance abuse itself was an important contributing factor to the visit. This was graded as direct (the patient seemed intoxicated), indirect (eg, social or financial problems due to substance abuse precipitated the visit), or no role at all. A direct or indirect relationship was significantly more prevalent in visits from patients with aggressive behaviors $(P<0.001$, OR 1.73, CI 1.41-2.12).

\section{Visit characteristics}

Over 30 reasons for a psychiatric referral were collapsed into nine logical groupings for analysis. The profile for visits with a current history of aggressive behaviors differed markedly from that of those without (Figure 3), primarily due to an increase in the number of referrals for behavioral dyscontrol and a decreased number for depression and anxiety-related disorders. Psychosocial stressors prior to the 


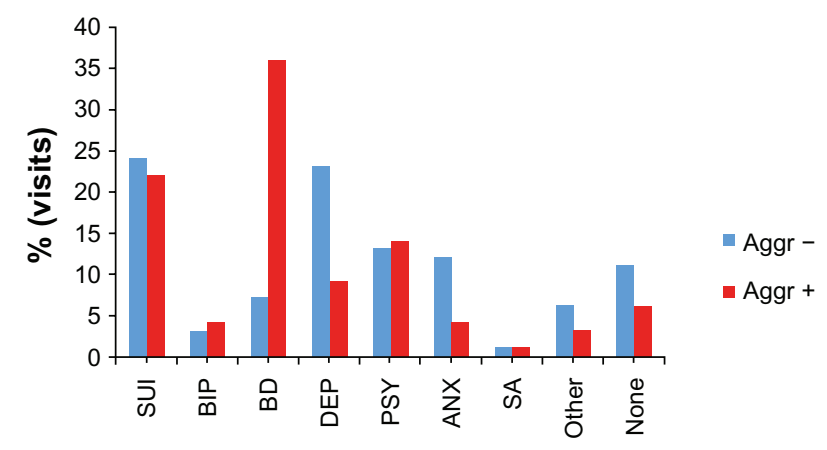

Figure 3 Profiles for the nine collapsed reasons for a psychiatric referral for visits with a current history of aggression $(n=1440)$ versus those without $(n=15,561)$. Adding the percentages of each of the individual blue or red columns totals $100 \%$. The profiles differed significantly (Pearson Chi-square $P<0.00 \mathrm{I}$ ).

Abbreviations: sui, suicidal ideation or acts; BIP, bipolar-related referral, eg, hypomania, mania; BD, behavioral dyscontrol; DEP, reason related to depression, eg, sadness; PSY, psychosis, hallucinations, bizarre ideation; ANX, eg, anxiety, nervousness, panic; $\mathrm{SA}$, any reason related to substance abuse; Other, eg, insomnia, side effects, legal opinion; none, no stated reason on the referral.

PES visit were reported in $76 \%$ (1127 of 1475 visits) of those marked by aggression and $72 \%$ (11,601 of 16,200 visits) of those without. The profiles of the psychosocial stressors differed significantly between visits with and visits without aggressive behaviors $(P<0.001$, Pearson chi-square). Relational ( $63 \%$ versus $48 \%$ ), legal ( $2 \%$ versus $1 \%$ ), and housingrelated ( $7 \%$ versus $6 \%)$ stressors were more frequent in visits with aggression, whereas the reverse was true for many of the remaining stressors, such as grief/loss ( $3 \%$ versus $6 \%)$, illness in self or significant other ( $6 \%$ versus $11 \%)$, employment-related ( $4 \%$ versus $9 \%$ ), financial (3\% versus $5 \%$ ), or other (12\% each). Current psychotropic medication was not found to differ in visits from patients with a current history of aggression $(n=1408)$ from those without $(n=17,303)$. However, compliance differed markedly, because visits from patients noncompliant with their medication were twice as likely to be marked by aggression $(P<0.001$, OR 2.15 , CI $1.81-2.55)$.

The diagnostic profiles of visits with and without aggression are shown in Figure 4. Personality, organic mental, substance abuse disorders and, to a slightly lesser extent, schizophrenia, were the diagnoses primarily observed in visits with aggression. Figure 5 shows the percentage of visits with aggression within each broad diagnostic category. In $44 \%$ (609 of 1389) of visits with a present history of aggression and 38\% (5554 of 14,463) of those without, a comorbid diagnosis was attributed. Substance abuse and personality disorders were the most frequent comorbid diagnoses (approximately $30 \%$ each), irrespective of the presence of aggression.

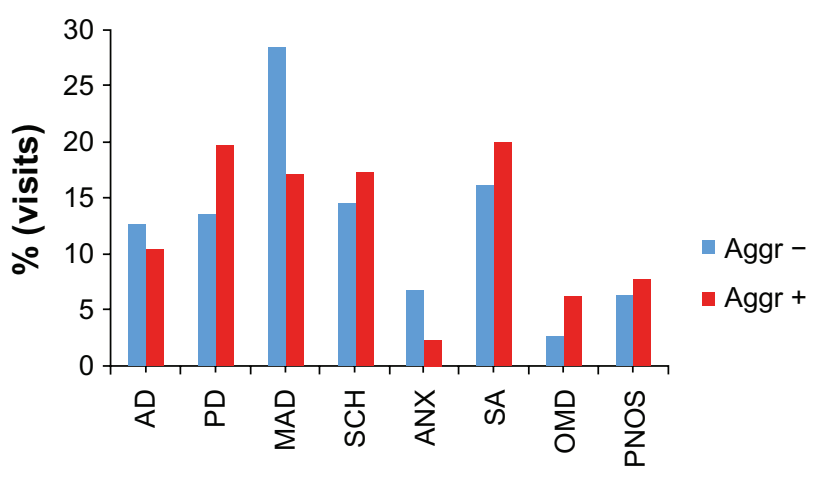

Figure 4 Diagnostic profiles of visits with a current history of aggression $(n=1389)$ versus those without $(n=14,463)$. Adding the percentages of each of the individual blue or red columns totals $100 \%$. The profiles differed significantly (Pearson Chisquare $P<0.001)$.

Abbreviations: AD, adjustment disorders; PD, personality disorders; MAD, major affective disorders, including bipolar disorders; SCH, schizophrenia; ANX, anxiety disorders; SA, substance abuse disorders; OMD, organic mental disorders; PNOS, psychoses not otherwise specified.

The outcome of the consultation process is shown in Figure 6. Noteworthy is the predominance of either observation or hospitalization in visits marked by aggression, compared with those without. Also, patients with visits marked by aggression $(n=1261)$ were three times more likely than those without $(n=13,674)$ to be placed under civil commitment in the PES following the psychiatric assessment $(P<0.001$, CI 2.66-3.59).

Finally, as previously reported, ${ }^{20}$ staff were asked to grade 16,821 visits $(14,821$ without and 1401 with aggression) qualitatively for both pertinence and urgency. About $71 \%$ of visits with aggression and $61 \%$ of visits without were judged pertinent and urgent, whereas $26 \%$ of visits with aggression and $17 \%$ of those without were judged pertinent but not urgent.

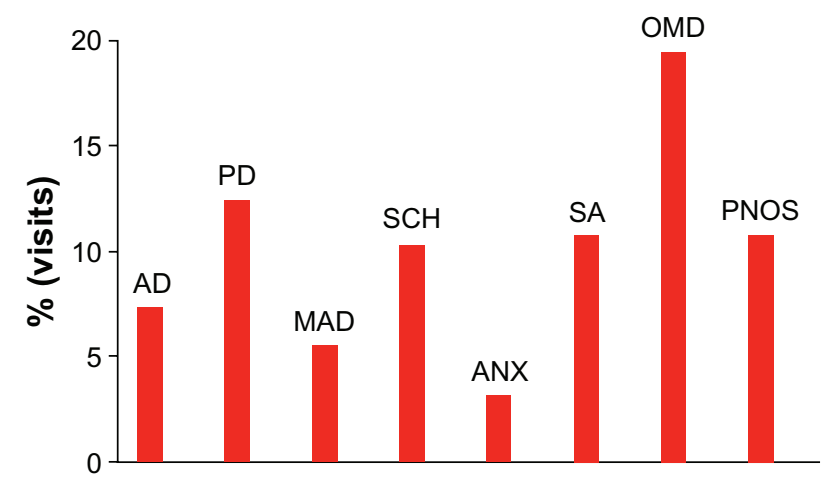

Figure 5 Percentage of visits with aggression $(n=1389)$ within each broad diagnostic category.

Abbreviations: $A D$, adjustment disorders; PD, personality disorders; MAD, major affective disorders, including bipolar disorders; $\mathrm{SCH}$, schizophrenia; ANX, anxiety disorders; SA, substance abuse disorders; OMD, organic mental disorders; PNOS, psychoses not otherwise specified. 


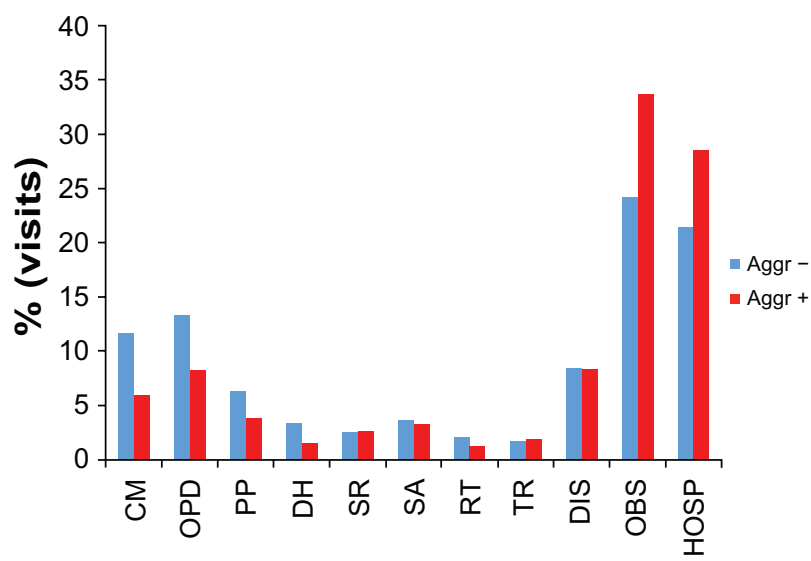

Figure 6 Outcome of visits with a current history of aggression $(n=1532)$ versus the outcome profile of those without a current history of aggression $(n=14,287)$. Adding the percentages of each of the individual blue or red columns totals $100 \%$. The profiles differed significantly (Pearson Chi-square $P<0.001$ ).

Abbreviations: Aggr-, without a current history of aggression; Aggr+, with a current history of aggression; CM, crisis management; OPD, outpatient follow-up; PP, general practitioner; DH, day hospital; SR, social resources; SA, detoxification centers; RT, refused treatment; TR, transfer to another PES; DIS, discharged without follow-up; OBS, observation in the PES; HOSP, hospitalization.

Approximately $10 \%$ of visits in each group were judged neither pertinent nor urgent. An assessment could not be made in $2 \%$ of visits in both groups. These profiles differed significantly (Pearson Chi-square $P<0.001$ ).

\section{Discussion}

Overall, aggressive behaviors in this study were found in $7.4 \%$ of all visits, a number in line with the few existing publications on this topic. ${ }^{10-12,22}$ However, noteworthy is that this percentage is most likely to be an underestimation because these behaviors were primarily assessed upon arrival to the PES, which is a major limitation of our data. The most coercive type of staff response, ie, physical restraint, was used in $28 \%$ of cases when aggression was current, which is a percentage comparable with the $24 \%$ reported by Mignon et al, ${ }^{23}$ within the first two hours of admission to the PES of agitated or violent patients. Approximately $50 \%$ of aggressive behaviors were dealt with by staff using verbal pacification. Verbal intervention was reported to be the most frequent alternative to physical restraints used in aggressive patients in a census of specialized and non-specialized emergency department and psychiatric emergency departments in the US..$^{24}$

In the present study, we found aggressive behaviors to be prevalent in all broad diagnostic categories, with the possible exception of anxiety disorders. However, anxiety disorders contributed little to the overall PES diagnostic profile (Figure 4) and had the least proportion of visits ("within group") marked by aggression of all diagnostic categories
(Figure 5). Organic mental disorders similarly contributed little to the overall PES diagnostic profile, but had an almost $20 \%$ proportion of "within group" visits marked by aggression. Active psychosis (eg, hallucinations, delusional thoughts) as a reason for a psychiatric referral was equally prevalent in visits with and without aggression. Overall, visits tagged with aggression were only marginally more comorbid than those without, and the two diagnostic categories most often reported as comorbidity, substance abuse, and personality disorders, were equally reported in visits without aggressive behaviors.

These results would seem to contrast with the oftenreported link between psychoses and aggressive behaviors in the inpatient setting ${ }^{1,6-9}$ and the results of Oster et al, ${ }^{10}$ who reported a moderate correlation with psychosis in the PES. However, the differences here may be more apparent than real. In the present study, we used broad diagnostic categories, rather than state-dependent symptoms. It is possible that creating a psychosis binary variable set (combining schizophrenia, psychosis not otherwise specified, and psychotic variants of the major affective disorders) would have better illustrated such a state effect. Furthermore, the PES, as a front-line system in mental health care delivery, is called upon to assess and, increasingly, to treat a very broad range of psychopathology. ${ }^{25,26}$ This broad range in itself would tend to dilute the importance of any one diagnostic category. Third, diagnostic stability may be a possible limiting factor in this study. Baca-Garcia et al, ${ }^{27}$ using a retrospective, semiadministrative database, found that diagnostic stability varied with diagnosis (best for schizophrenia, least for personality disorders) and setting (best in the inpatient, least in the outpatient, intermediate in the PES setting). Using purely administrative databases, the PES has fared worse.$^{28}$ Although we used a prospective, non-administrative database combined with a rigorous methodological approach in order to reduce diagnostic uncertainty, such a phenomenon may remain a possible confounding factor in our study.

Age was found to be a marginal explanatory variable, in contrast with previous reports. ${ }^{29}$ One possible explanation might be that most PES visits are made by those aged 44 years and younger,,$^{30-34}$ making it difficult to separate young aggressive patients from an overall young population. Many sociodemographic variables were indeed significantly more likely to be associated with visits marked by aggression. However, on the whole, many of these variables were somewhat predictable. Visits from single, poorly educated, or unemployed patients and those with a past history of illegal activities, were typically associated with aggressive 
behaviors. A gender difference favoring males was observed overall, and was also observed within each broad diagnostic category (data not shown). That being said, a gender difference in almost all types of violent behaviors is well documented by many national statistical agencies. ${ }^{35,36}$

Arrival in the evening or night has been associated with a greater risk of aggressive behavior in the medical emergency department and, in at least one report, the PES. ${ }^{37-39}$ We could not confirm this finding in the present study, although we did find a tendency for visits with aggression to be within the noon to 8 pm time slot. Other PES-specific variables such as frequency of use, were not associated with a higher risk of aggressive behaviors while others were, eg, the type of arrival. Involuntary arrivals, regardless of referral source, markedly increased the risk of aggression. In this same line, patients brought to the PES by the police, judiciary, or their landlords (apartment, group home, residence) showed significantly more aggressive behaviors than those who came alone. Behavioral dyscontrol and suicidal ideation (or acts) accounted for nearly $60 \%$ of all reasons for a referral in visits with aggression. Given the importance of substance abuse in visits with aggression (prior history of, as a primary or as a comorbid diagnosis), there were, paradoxically, few referrals specifically for this reason. Rather, referring emergency department physicians appeared to focus on factors such as poor impulse control and suicidal ideation, which are observed in almost $50 \%$ of patients with alcohol dependence. ${ }^{40}$ However, interestingly, the psychiatric nursing staff in these services subjectively graded alcohol or drug abuse as being an important direct and/or indirect contributing factor to many of these visits.

Overall, the sociodemographic and clinical picture is one that evokes a downward-drift type of patient, more typically a younger male, unemployed, single or separated, with a history of substance abuse. Such a patient is more likely to be brought in involuntarily and/or by the police, the judiciary or by a landlord, and to report a stress prior to the visit that is interpersonal in nature. If taking medication, the patient is likely to be noncompliant, and most often referred for behavioral dyscontrol, regardless of the presence or absence of an underlying active or chronic psychosis (although an underlying psychosis is often present in such patients).

The cost of aggressive behaviors in the PES does not appear to be only psychological in nature. For instance, the outcome varied significantly with the presence of aggression. Visits marked by aggressive behaviors were more likely to result in either observation in the PES or hospitalization. Indeed, almost all discharge variables were proportionally lower in visits marked by aggression. Furthermore, the latter also more frequently resulted in civil commitment in the PES, which is an additional financial burden.

\section{Conclusion}

Care must be taken when generalizing from what are largely regional data, because they may not always accurately reflect national trends. Nevertheless, our results do suggest that many clinical and demographic state-independent variables are associated with aggressive behaviors in the PES. Although their sum may not add up to a specific patient profile, they can nevertheless be useful in service planning, being easily integrated alongside state-dependent rating scales in a triage and/or observation instrument for daily use by the PES.

\section{Acknowledgment}

This work was partly supported by Valorisation Recherche Québec.

\section{Disclosure}

This research was presented at the 60th annual conference of the Canadian Psychiatric Association in Toronto, Canada, on October 8, 2010. The authors declare that they have no competing interests in this work.

\section{References}

1. Flannery RB Jr, Farley E, Tierney T, Walker AP. Characteristics of assaultive psychiatric patients: 20 -year analysis of the assaultive staff action program (ASAP). Psychiatr Q. September 8, 2010. [Epub ahead of print].

2. Lemelin L, Bonin JP, Duquette A. Workplace violence reported by Canadian nurses. Can J Nurs Res. 2009;41(3):152-167.

3. Lipscomb JA, Love CC. Violence toward health care workers: An emerging occupational hazard. AAOHN J. 1992;40(5):219-228.

4. McNiel DE, Binder RL. Psychiatric emergency service use and homelessness, mental disorder, and violence. Psychiatr Serv. 2005;56(6): 699-704.

5. Foust D, Rhee KJ. The incidence of battery in an urban emergency department. Ann Emerg Med. 1993;22(3):583-585.

6. Abderhalden C, Needham I, Dassen T, Halfens R, Haug HJ, Fischer JE. Structured risk assessment and violence in acute psychiatric wards: Randomised controlled trial. Br J Psychiatry. 2008;193(1):44-50.

7. Abeyasinghe R, Jayasekera R. Violence in a general hospital psychiatry unit for men. Ceylon Med J. 2003;48(2):45-47.

8. Casper ES, Donaldson B. Subgroups in the population of frequent users of inpatient services. Hosp Community Psychiatry. 1990;41(2): 189-191.

9. Kennedy J, Bresler S, Whitaker A, Masterson B. Assessing violence risk in psychiatric inpatients: Useful tools. Psychiatr Times. 2007;24(8) Available from: http://www.psychiatrictimes.com/display/ article/10168/54696. Accessed November 15, 2010.

10. Oster A, Bernbaum S, Patten S. Determinants of violence in the psychiatric emergency service. CMAJ. 2001;164(1):32-33.

11. Skokol AE, Karasu TB. Emergency psychiatry and the assaultive patient. Am J Psychiatry. 1978;135:202-205.

12. Boudreaux ED, Allen MH, Claassen C, et al. The Psychiatric Emergency Research Collaboration-01: Methods and results. Gen Hosp Psychiatry. 2009;31(6):515-522. 
13. Beck JC, White KA, Gage B. Emergency psychiatric assessment of violence. Am J Psychiatry. 1991;148(11):1562-1565.

14. Dhossche DM. Aggression and recent substance abuse: Absence of association in psychiatric emergency room patients. Compr Psychiatry. 1999;40(5):343-346.

15. McNiel DE, Myers RS, Zeiner HK, Wolfe HL, Hatcher C. The role of violence in decisions about hospitalization from the psychiatric emergency room. Am J Psychiatry. 1992;149(2):207-212.

16. Chaput Y, Lebel MJ. Demographic and clinical profiles of patients who make multiple visits to psychiatric ermegency services. Psychiatr Serv. 2007;58(3):335-341.

17. Chaput YJ, Lebel MJ. An examination of the temporal and geographical patterns of psychiatric emergency service use by multiple visit patients as a means for their early detection. BMC Psychiatry. 2007;7(1):60.

18. Catalano R, McConnell W, Forster P, McFarland B, Thornton D. Psychiatric emergency services and the system of care. Psychiatr Serv. 2003;54(3):351-355.

19. Almvik R, Woods P. Short-term risk prediction: The Broset Violence Checklist. J Psychiatr Ment Health Nurs. 2003;10(2):236-238.

20. Chaput Y, Paradis M, Beaulieu L, Labonte E. A qualitative study of a psychiatric emergency. Int J Ment Health Syst. 2008;2(1):9.

21. Pasic J, Russo J, Roy-Byrne P. High utilizers of psychiatric emergency services. Psychiatr Serv. 2005;56(6):678-684.

22. Saliou V, Fichelle A, McLoughlin M, Thauvin I, Lejoyeux M. Psychiatric disorders among patients admitted to a French medical emergency service. Gen Hosp Psychiatry. 2005;27(4):263-268.

23. Migon MN, Coutinho ES, Huf G, Adams CE, Cunha GM, Allen MH. Factors associated with the use of physical restraints for agitated patients in psychiatric emergency rooms. Gen Hosp Psychiatry. 2008;30(3): 263-268.

24. Downey LV, Zun LS, Gonzales SJ. Frequency of alternative to restraints and seclusion and uses of agitation reduction techniques in the emergency department. Gen Hosp Psychiatry. 2007;29(6):470-474.

25. Pelland ME, Marchand A, Lessard MJ, et al. Efficacy of 2 interventions for panic disorder in patients presenting to the ED with chest pain. Am J Emerg Med. September 24, 2010. [Epub ahead of print].

26. Milner KK, Barry KL, Blow FC, Welsh D. Brief interventions for patients presenting to the psychiatric emergency service (PES) with major mental illnesses and at-risk drinking. Community Ment Health J. 2010;46(2):149-155.

27. Baca-Garcia E, Perez-Rodriguez MM, Basurte-Villamor I, et al. Diagnostic stability of psychiatric disorders in clinical practice. $\mathrm{Br} \mathrm{J}$ Psychiatry. 2007;190:210-216.
28. Folsom DP, Lindamer L, Montross LP, et al. Diagnostic variability for schizophrenia and major depression in a large public mental health care system dataset. Psychiatry Res. 2006;144(2-3):167-175.

29. Flannery RB Jr, Juliano J, Cronin S, Walker AP. Characteristics of assaultive psychiatric patients: Fifteen-year analysis of the Assaulted Staff Action Program (ASAP). Psychiatr Q. 2006;77(3):239-249.

30. Adityanjee, Mohan D, Wig NN. Determinants of emergency room visits for psychological problems in a general hospital. Int J Soc Psychiatry. 1988;34(1):25-30.

31. Bauer SF, Balter L. Emergency psychiatric patients in a municipal hospital. Demographic, clinic clinical and dispositional characteristics. Psychiatr Q. 1971;45(3):382-393.

32. Claassen CA, Hughes CW, Gilfillan S, et al. Toward a redefinition of psychiatric emergency. Health Serv Res. 2000;35(3):735-754.

33. Claassen CA, Michael Kashner T, Gilfillan SK, Larkin GL, John Rush A. Psychiatric emergency service use after implementation of managed care in a public mental health system. Psychiatr Serv. 2005;56(6): 691-698.

34. Bruffaerts R, Sabbe M, Demyttenaere K. Who visits the psychiatric emergency room for the first time? Soc Psychiatry Psychiatr Epidemiol. 2006;41(7):580-586.

35. Statistics Canada. Canadian crime statistics 2003. Available from: http://www.statcan.gc.ca/pub/85-205-x2003000-eng.pdf. Accessed November 15, 2010.

36. US Department of Justice. Crime in the United States. Available from: http://ww2.fbi.gov/ucr/cius2007/data/table_22.html. Accessed November 15, 2010.

37. Cusimano M, Marshall S, Rinner C, Jiang D, Chipman M. Patterns of urban violent injury: A spatio-temporal analysis. PLoS One. 2010;5(1):e8669.

38. Brookes JG, Dunn RJ. The incidence, severity and nature of violent incidents in the emergency department. Emergency Medicine. 1997; 9(1):5-9.

39. Godemann F, Uhlemann H, Hauth I. Psychiatric emergency services inpatient admissions during the night. German Journal of Psychiatry. 2006;9:128-132.

40. Conner K, Hesselbrock VM, Meldrum SC, et al. Transitions to, and correlates of, suicidal ideation, plans, and unplanned and planned suicide attempts among 3,729 men and women with alcohol dependence. J Stud Alcohol Drugs. 2007;68:654-662.
Open Access Emergency Medicine

\section{Publish your work in this journal}

Open Access Emergency Medicine is an international, peer-reviewed, open access journal publishing original research, reports, editorials, reviews and commentaries on all aspects of emergency medicine. The manuscript management system is completely online and includes a very quick and fair peer-review system, which is all easy to use.

\section{Dovepress}

Visit http://www.dovepress.com/testimonials.php to read real quotes from published authors. 